\title{
OPTIMUM SAMPLING SCHEME FOR CHARACTERIZATION OF MINE TAILINGS
}

\section{P. Debba}

\author{
The Council for Scientific and Industrial \\ Research (CSIR) \\ CSIR Built Environment \\ P. O. Box 395, 0001, South Africa \\ email: pdebba@csir.co.za
}

\author{
E.J.M. Carranza, A. Stein, F.D. van der Meer \\ International Institute for Geo-Information \\ Science and Earth Observation (ITC) \\ Hengelosestraat 99, P. O. Box 6, 7500AA \\ Enschede, The Netherlands
}

\begin{abstract}
The paper describes a novice method for sampling geochemicals to characterize mine tailings. We model the spatial relationships between a multi-element signature and, as covariates, abundance estimates of secondary iron-bearing minerals in mine tailings dumps. The covariates of interest, are readily, but less accurately obtainable by using airborne hyperspectral data and estimated through spectral unmixing. Via simulated annealing an optimal prospective sampling scheme for a new unvisited area is derived based on the variogram model of a previously sampled area.
\end{abstract}

Index Terms - Sampling, mine tailings, remote sensing, hyperspectral, unmixing, external drift kriging, variogram

\section{BACKGROUND AND OBJECTIVE}

Geochemical characterization of mine waste impoundments is important for rehabilitation, or for remediation, to protect the surrounding environment and ecosystems. For effective geochemical characterization, this would entail surface (to subsurface) sampling, which could be labor or cost intensive, especially if not properly planned. Metals in mine waste impoundments are usually hosted by acidgenerating sulphide-rich minerals, for e.g., pyrite, pyrrhotite, or adsorbed onto surfaces of weathering products of such sulphide-rich minerals. Unfortunately, such minerals are difficult to detect or identify by using current remote sensing techniques including multispectral or even hyperspectral data. It has been shown, however, that certain sulphide-rich minerals, particularly pyrite, weathers to a series of iron-bearing sulfates, hydroxides and oxides [1]. Such secondary iron-bearing sulfates/hydroxides/oxides have diagnostic spectral features [2], which enable their detection or identification with analytical techniques using hyperspectral data.

Debba et. al. (2006) [3] demonstrated the potential of using hyperspectral data to estimate abundances of spectrally similar iron-bearing sulfates/hydroxides/oxides. It has also been shown that heavy metal contamination in soils can be quantified using reflectance spectroscopy [4]. Thus, remote sensing technology potentially provides an indirect tool for surface characterization of mine waste impoundments with oxidizing sulphide-rich materials; namely, for mapping spatial distributions of secondary iron-bearing sulfates/hydroxides/oxides and thereby modeling the spatial distribution of heavy metals. Hence, given a model of spatial distribution of secondary iron-bearing oxides/hydroxides, the problem is how to design a sampling scheme that would adequately capture the spatial distribution of certain groups of metals.
In this paper, a prospective sampling scheme is derived for nearby unsampled areas based on the variogram model of the adjacent sampled area. The present case study area is in the RecskLahóca copper mining area in Hungary. The Recsk-Lahóca mining area is situated in the Mátra Mountains, about $110 \mathrm{~km}$ northeast of Budapest, Hungary. Mining of ore deposits in the Recsk-Lahóca area resulted in the exposure of sulphide bearing-rocks to surface water and atmospheric oxygen, which accelerate oxidation, leaching and release of metals and acidity. Mine tailings and waste rock dumps resulting from the two-century mining of copper and gold are present in the area.

\section{DATA}

In this study, a subset of the Digital Airborne Imaging Spectrometer (DAIS-7915) is used. The resulting data is a 79 channel hyperspectral image, acquired over Recsk on 18th August 2002 and is shown in Figure 1 at $5 \mathrm{~m}$ nominal resolution on the ground. Not all 79 channels were useful as many channels were too noisy and could not be corrected efficiently. Fortunately, the first 32 channels, spectral range 406-1035 nm, where iron-bearing oxides/hydroxides/sulphates have diagnostic features were found useful for this study.

Samples from the tailings (Figure 1) were collected a few minutes shortly after collection of the DAIS hyperspectral data. Fiftythree samples were collected in the East Tails and 44 in the West tails. Samples of tailings were collected at $10 \mathrm{~m} \times 10 \mathrm{~m}$ grid points in portions of the tailings dumps with almost no vegetation cover within $3 \mathrm{~m}$ radius. Portions of the tailings dumps, close to the active stream have steep slopes and were not sampled. Samples were collected from the top surface of the tailings and were analyzed in the laboratory. Concentrations of $\mathrm{As}, \mathrm{Cd}, \mathrm{Cu}, \mathrm{Fe}, \mathrm{Mn}, \mathrm{Ni}, \mathrm{Pb}, \mathrm{Sb}$ and $\mathrm{Zn}$ in the decomposed samples were determined using the ICP-AES analyzer.

\section{METHODOLOGY}

The East Tails and the West Tails have different geochemical characteristics, hence it was decided to split the data into two sets. The small stream between the East Tails and the West Tails provides a natural boundary to do so. Data from either sub-area are used to model a relationship between heavy metal associations and relative abundances of secondary iron-bearing minerals. The latter data are derived from spectral unmixing of hyperspectral data. A model relationship between heavy metal associations and mineral abundances 


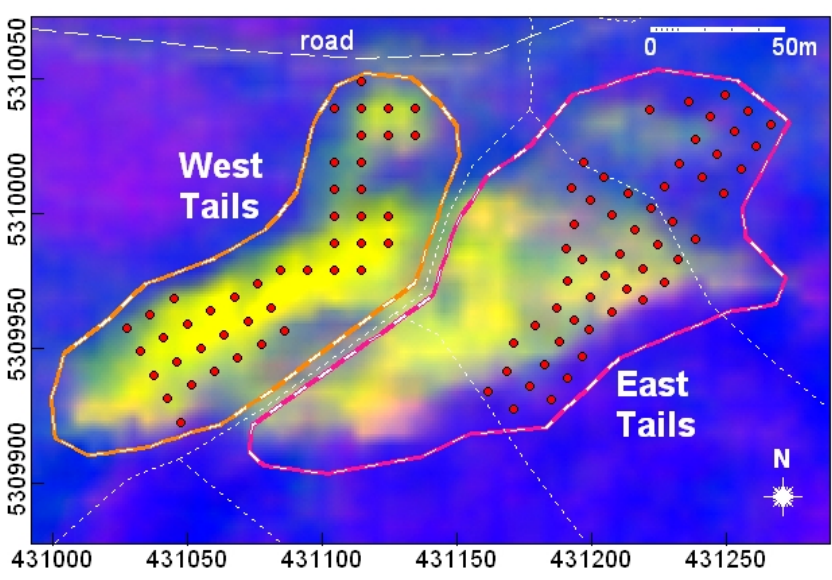

Fig. 1. The "East Tails" and the "West Tails" shown in a color composite image of the DAIS data. Ratios of ch17 to ch28 (representing ferrihydrite reflectance and absorption peaks) was used as red band, ch13 to ch 25 (representing jarosite reflectance and absorption peaks) was used as green band and ch32 to ch1 (representing noniron-bearing minerals) was used as blue band. Red dots are locations of mine tailings samples. Short dashed lines indicates drainage lines of either active or non-active streams.

in one sub-area is then used as basis for optimal sampling design in the other sub-area. Division of the area and the data thus provides calibration analysis and prediction/validation analysis for optimal sampling design.

\subsection{Estimation of mineral abundance}

Spectral unmixing of hyperspectral data was performed to estimate relative abundance or proportion, per $5 \mathrm{~m}$ pixel, of secondary ironbearing minerals, with which metals in the mine tailings could be associated. Spectral unmixing is a deconvolution process for estimating proportional contributions of each end-member to spectra. Debba et. al. (2006) [3] suggested that better abundance estimates are obtained if materials, not necessarily of interest but are probably present, and contribute to a pixel spectrum is also included in an endmember set for spectral unmixing. Accordingly, copiapite, jarosite, goethite, ferrihydrite, hematite, kaolinite, anhydrite, gypsum, quartz, and tumbleweed (grass) was considered to consist the end-member set. Abundance estimates were determined according to the method of [3], which involves minimization of variance of the differences between the first derivative of an estimated spectrum and the first derivative of an actual spectrum after smoothing the spectra.

\subsection{Modeling of heavy metal associations}

Concentrations of several metals in soils can be estimated using reflectance spectroscopy [4]. In addition, geochemical sampling addresses a suite of metals, which reflect intrinsic processes in a system, like a mine tailings dump. It was thus decided to model a heavy metals association reflecting scavenging of metals by secondary iron-bearing minerals in the mine tailings dumps. A factor component analysis with varimax rotation was performed on the logarithmic-transformed heavy metal concentrations to obtain the heavy metal association of interest. The scores of FA2E and FA2W are then linearly transformed to $[0,1]$, for numerical compatibility with the mineral abundance estimates, and labeled FA2ET and FA2WT, respectively.

\subsection{Kriging with external drift}

Kriging with external drift is applicable to estimate primary variables of interest, which are practically measurable at only few sample sites, based on linearly related ancillary variables, which are measurable at much higher sampling density than the primary variables. Wackernagel (1998) [5] suggests that such ancillary variables can be incorporated into a kriging system as external drift functions. Kriging with external drift is ideal if a primary variable could be measured more precisely and practically at a few locations, whereas possibly less accurate measurements of linearly related ancillary variables are available everywhere in the spatial domain. The present case study applies kriging with external drift to model a relationship between heavy metal association of interest and metal-savenging minerals in the mine tailings dumps. The distribution of heavy metal associations, represented as factor scores, were used as the primary variable of interest and is based on field sampling. The relative abundances of metal-scavenging iron-bearing minerals, which were obtained from hyperspectral data, are the ancillary variables.

For the modeling, consider $\mathbf{x} \in \mathbf{A} \subset \mathbb{R}^{2}$ to be a generic data location $\left(x_{u}, x_{v}\right)$ in 2-dimensional Euclidean space and suppose the domain $Z(\mathbf{x})$ at spatial location $\mathbf{x}$ is a random quantity. The multivariate random field $\{Z(\mathbf{x}): \mathbf{x} \in \mathbf{A}\}$, is generated by letting $\mathbf{x}$ vary over index set $\mathbf{A} \subset \mathbb{R}^{2}$. A realization of this is denoted by $\{z(\mathbf{x}): \mathbf{x} \in \mathbf{A}\}$.

The semi-variogram $\gamma(h)$ is defined as half the average squared difference between values separated by a given lag $h$, where $h$ is a vector in both distance and direction, that is,

$$
\gamma(h)=\frac{1}{2} E[Z(\mathbf{x})-Z(\mathbf{x}+h)]^{2} .
$$

The experimental semi-variogram $\gamma^{\star}(h)$ may be obtained from $\kappa=1,2, \ldots, P(h)$ pairs of observations $\left\{z\left(\mathbf{x}_{\kappa}\right), z\left(\mathbf{x}_{\kappa}+h\right)\right\}$ at locations $\left\{\mathbf{x}_{\kappa}, \mathbf{x}_{\kappa}+h\right\}$, as:

$$
\gamma^{\star}(h)=\frac{1}{2 \cdot P(h)} \sum_{\kappa=1}^{P(h)}\left[z\left(\mathbf{x}_{\kappa}\right)-z\left(\mathbf{x}_{\kappa}+h\right)\right]^{2} .
$$

Suppose that precise measurements are available for a primary variable $Z(\mathbf{x})$ with $n_{B}$ observations, which is assumed to be a second order random function with known covariance function $C(h)$, hence the variogram $\gamma(h)=C(h) / C(0)$ is assumed to be known. The $k$ ancillary variables represented as regionalized variables $y_{i}(\mathbf{x}), i=1, \ldots, k$ with $n_{A}$ observations, are less accurate measurements covering the whole domain $\mathbf{A}$ at small scale and are considered as deterministic. The values $\left\{y_{i}(\mathbf{x})\right\}$ needs to be known at all locations $\mathbf{x}_{\alpha}$ of the samples as well as at the nodes of the estimation grid.

Since $Z(\mathbf{x})$ and the set of $\left\{y_{i}(\mathbf{x})\right\}$ are two ways of expressing the same phenomenon, assume that $Z(\mathbf{x})$ is on average equal to a linear function of the set of $\left\{y_{i}(\mathbf{x})\right\}$ up to a constant $b_{0}$ and coefficients $b_{i}, i=1, \ldots, k$,

$$
E[Z(\mathbf{x})]=b_{0}+\sum_{i=1}^{k} b_{i} \cdot y_{i}(\mathbf{x})=\sum_{i=0}^{k} b_{i} \cdot y_{i}(\mathbf{x}),
$$

where $y_{0}(\mathbf{x})=1$. The method of merging both sources of information uses $\left\{y_{i}(\mathbf{x})\right\}$ as an external drift function for the estimation of 
$Z(\mathbf{x})$. The drift of $Z(\mathbf{x})$ is defined externally through the ancillary variables $\left\{y_{i}(\mathbf{x})\right\}$ rather than some smooth version of $Z(\mathbf{x})$ itself, [6]. then

Assuming $Z(\mathbf{x})$ is a second order stationary random function,

$$
Z^{*}\left(\mathbf{x}_{0}\right)=\sum_{\alpha=1}^{n_{A}} \lambda_{\alpha} Z\left(\mathbf{x}_{\alpha}\right)
$$

where $\lambda_{\alpha}$ denotes the weight of the $\alpha$ th observation and is constraint to unit sum.

In estimating the external drift coefficients, the following conditions,

$$
\sum_{\alpha=1}^{n_{A}} \lambda_{\alpha} y_{i}\left(\mathbf{x}_{\alpha}\right)=y_{i}\left(\mathbf{x}_{0}\right), \quad i=1, \ldots, k,
$$

are added to the kriging system independently of the inference of the covariance function, hence the term "external". The kriging variance can then be written as

$$
\sigma_{\mathrm{KED}}^{2}\left(\mathbf{x}_{0}\right)=\operatorname{Var}\left[Z\left(\mathbf{x}_{0}\right)-Z^{*}\left(\mathbf{x}_{0}\right)\right] .
$$

The only factor influencing the kriging variance are the variogram $\gamma(h)$, the number of observations $n_{A}$, the sampling locations $\mathbf{x}_{\alpha}$ and the location $\mathbf{x}_{0}$. This means that the kriging variance does not depend on the observations themselves, but rather only on their relative spacing. The advantage is that in can be used to optimize sampling schemes in advance of data collection.

In this paper, the location and the covariates as external drift were used to estimate the heavy metal concentration,

$$
\begin{aligned}
E[Z(\mathbf{x})]= & b_{0}+b_{1} \cdot x_{u}+b_{2} \cdot x_{v}+b_{3} \cdot \operatorname{GOE}(\mathbf{x}) \\
& +b_{4} \cdot \operatorname{JAR}(\mathbf{x})+b_{5} \cdot \operatorname{FER}(\mathbf{x})+b_{6} \cdot \operatorname{HEM}(\mathbf{x}) \\
& +b_{7} \cdot \operatorname{KAO}(\mathbf{x})+b_{8} \cdot \operatorname{COP}(\mathbf{x})
\end{aligned}
$$

namely, a first order polynomial on the coordinates and the abundance estimates of the metal-scavenging minerals. In Equation 7, GOE is abundance estimates for goethite, JAR is for jarosite, FER is for ferrihydrite, HEM is for hematite, KAO is for kaolinite, and COP is for copiapite.

\subsection{Sampling scheme optimization}

For a two-dimensional area $\mathbf{A}$, let the collection of all possible sampling schemes with $n$ observations on $\mathbf{A}$ be denoted by $\mathbf{S}^{n}$. A fitness function $\phi\left(\mathbf{S}^{n}\right): \mathbf{S}^{n} \rightarrow \mathbb{R}^{+}$is minimized through simulated annealing. In terms of sampling, the initial step in simulated annealing is to randomly select a set of points in $\mathbf{A}$. A new point in $\mathbf{A}$ is then randomly selected and replaces a randomly selected old point from the current collection. This replacement occurs, based on a probabilistic criterion, if the fitness function decreases and if the fitness function increases. Hence, the process allows inferior moves. Initially, the probabilistic criterion is high, allowing a large probability of inferior moves. A parameter in the annealing process is then reduced by a factor of 0.95 at each successive step, thereby decreasing the probability of accepting inferior moves until the process stabilizes. The final solution is independent of the initial random selection of points as the process reaches the global optimum.

A variogram model for a previously sampled area can be used to estimate $\sigma^{2}(\mathbf{x})$ (Equation 6) in an unvisited area, if the latter is considered to have similar, if not the same, spatial characteristics as the former. In this case, estimates of $\sigma^{2}(\mathbf{x})$ in the unvisited area can be used to derive the optimal prospective sampling scheme. The optimization procedure by simulated annealing is then performed by application of a criterion called the Mean Kriging Variance with External Drift (MKVED), the fitness function of which is defined as

$$
\phi_{\mathrm{MKVED}}(\mathbf{S})=\frac{1}{n_{\mathbf{A}}} \sum_{j=1}^{n_{\mathbf{A}}} \sigma_{\mathrm{KED}}^{2}\left(\mathbf{x}_{\mathbf{A}, j} \mid \mathbf{S}\right),
$$

where $n_{\mathbf{A}}$ is the number of raster nodes for which data for each of the covariates are available. The MKVED-criterion is ideal for deriving optimal prospective sampling schemes, since it does not depend on the actual data for the primary variable. It depends, however, on a covariance function, the spatial configuration of sampling locations and data locations of the covariates (Equation 6). Hence, the MKVED-criterion is proposed to derive the optimal prospective sampling scheme in an unvisited area based on a relevant model from a previously sampled area.

\section{RESULTS OF OPTIMAL PROSPECTIVE SAMPLING SCHEMES}

By way of simulated annealing, a prospective sampling scheme for the West Tails is derived based on a model for the East Tails. As an illustration, it was decided to derive a prospective sampling scheme having 30 samples in the West Tails using the 53 samples from the East Tails. The exponential variogram was estimated with the data from the East Tails and is shown in Figure 2(a). To verify that this variogram is also appropriate for the West Tails, the East and West Tails data were combined and the variogram is shown in Figure 2(b). The similarity of the two variograms indicate that the variogram for the East Tails could be appropriate for modeling the West Tails.

Variogram model for the East Tails was applied to the West Tails data to derive a prospective sampling scheme via simulated annealing to minimize Equation 8. The resulting prospective sampling scheme, with 30 samples for the West Tails, is shown in Figure 3. The optimal sampling scheme constructed using the kriging external drift variance approach are spread over the West Tails region while retaining some close pairs of samples. These close pair samples are to improve the estimation of the variogram model. The mean kriging with external drift variance for the West Tails, using the combined East and West Tails sampling data, as illustrated in Figure 1 , is $6.8 \times 10^{-4}$ for the West Tails. This mean kriging variance was approximately the same when either of the two variograms was used. The optimal sampling scheme resulted in a mean kriging with external drift variance for the West Tails of $3.3 \times 10^{-4}$ using the variogram derived from the East Tails data. This indicates that the optimal sampling scheme contains samples that reduces the mean kriging with external drift variance for the previously designed grid sampling scheme in the West Tails.

\section{DISCUSSION AND CONCLUSIONS}

Surface characterization of mine tailings could provide essential information for protection of surrounding ecosystems. Planning where tailings samples should be collected is therefore a crucial task. This is so because spatial distributions of undesirable heavy metals in mine tailings must be determined accurately. This study has demonstrated usefulness of airborne hyperspectral data to support optimization of sampling schemes for surface characterization of mine tailings.

Analysis of hyperspectral data can yield information about spatial distributions of secondary iron-bearing and clay minerals associated with weathering of pyrite-rich mine wastes. Heavy metals 


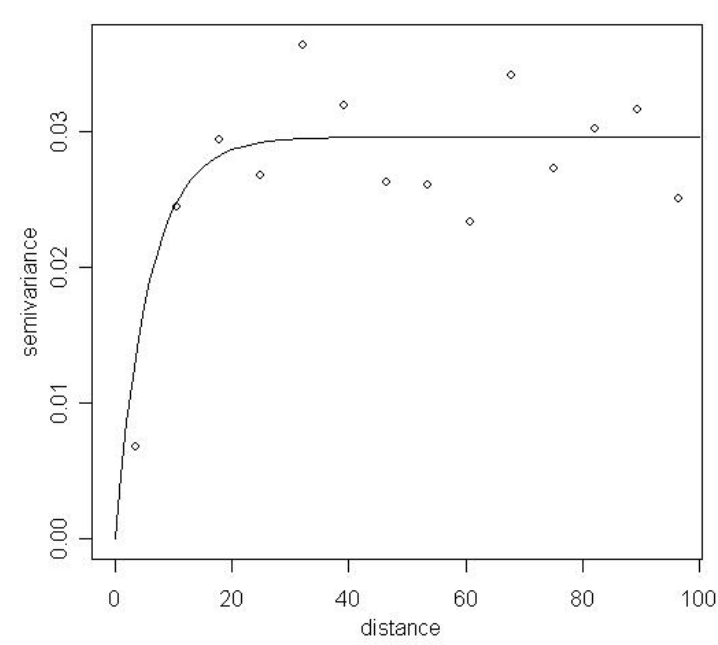

(a) East Tails

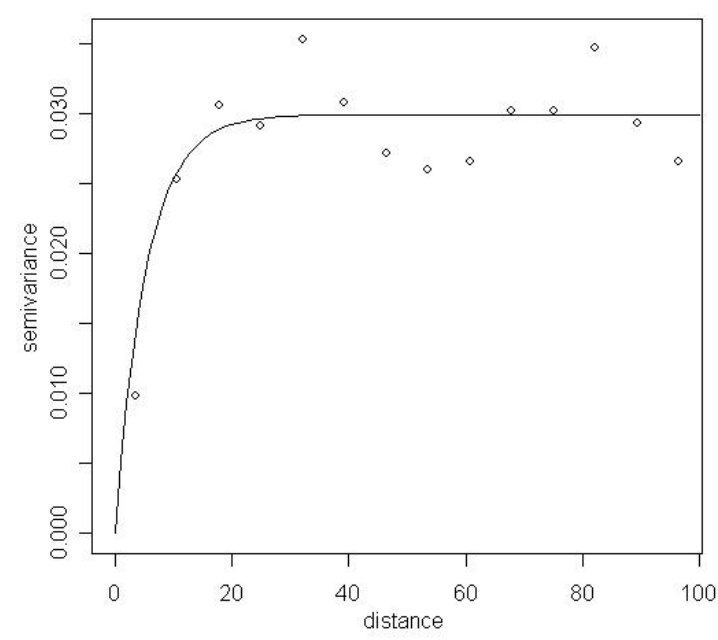

(b) East and West Tails combined

Fig. 2. The exponential variogram for the East Tails, combined East $\&$ West Tails.

usually reside in such secondary minerals. Nevertheless, field samples are necessary to model spatial relationships between heavy metals and secondary minerals in mine tailings. The data collected in an orientation grid sampling program must allow determination of (a) spatial distributions of heavy metals, (b) spatial distributions of secondary metal-scavenging minerals, (c) spatial relationships between heavy metals and secondary metal-scavenging minerals, and (d) a variogram model of heavy metal associations due to metalscavenging minerals. These four types of spatial information are essential to optimize a prospective sampling scheme to be carried out during a main sampling program, especially for large mine tailings.

This study has shown that spatial relationships between heavy metals and metal-scavenging minerals can be modeled adequately by kriging with external drift. The kriging variance, being dependent only on the variogram, the spatial configuration of the sampling locations and the data locations, could then be used to derive optimal sampling schemes. In conclusion, the use of secondary information in designing optimal sampling schemes was also illustrated. Often

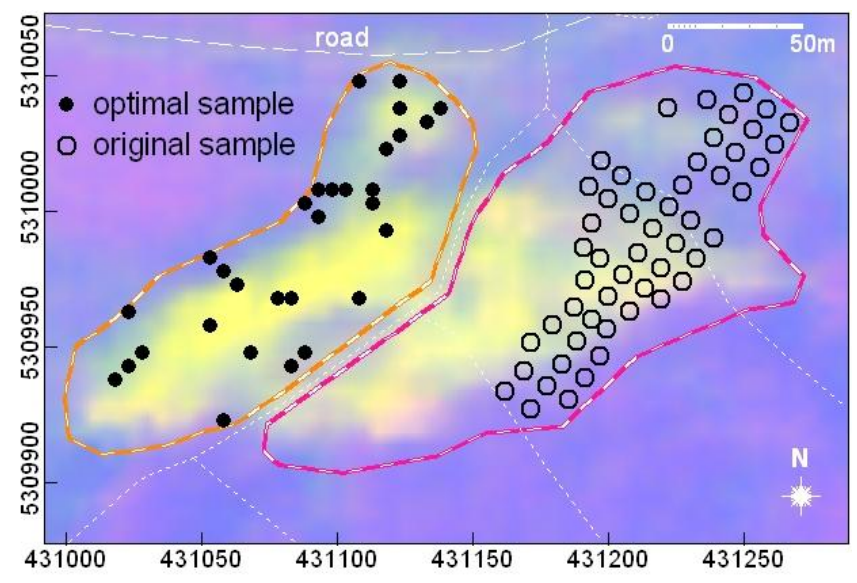

Fig. 3. Prospective optimal sampling scheme in the West Tails using East Tails samples.

these secondary information can be achieved at a relatively low cost and available over a greater region. These are the primary reasons for incorporating this information into the sampling design. Optimized sampling schemes using the mean kriging with external drift variance will result in sampling schemes that explicitly take into account the nature of spatial dependency of the data and together with hyperspectral data can be used to design sampling schemes in nearby unexplored areas.

\section{REFERENCES}

[1] G. A. Swayze, K. S. Smith, R. N. Clark, S. J. Sutley, R. M. Pearson, J. S. Vance, P. L. Hageman, P. H. Briggs, A. L. Meier, M. J. Singleton, and S. Roth, "Using imaging spectroscopy to map acidic mine waste," Environmental Science and Technology, vol. 34, pp. 47-54, 2000.

[2] J. K. Crowley, D. E. Williams, J. M. Hammarstrom, N. Piatak, I. M. Chou, and J. C. Mars, "Spectral reflectance properties $(0.4-2.5 \mu \mathrm{m})$ of secondary Fe-oxide, Fe-hydroxide, and Fe-sulphate-hydrate minerals associated with sulphide-bearing mine wastes," Geochemistry: Exploration, Environment, Analysis, vol. 3, no. 2, pp. 219-228, 2003.

[3] P. Debba, E. J. M. Carranza, F. D. van der Meer, and A. Stein, "Abundance estimation of spectrally similar materials by using derivatives in simulated annealing," IEEE Geoscience and Remote Sensing, vol. 44, no. 12, pp. 3649-3658, 2006.

[4] T. Kemper and S. Sommer, "Estimate of heavy metal contamination in soils after a mining accident using reflectance spectroscopy," Environmental Science and Technology, vol. 36, no. 12, pp. 2742-2747, 2002.

[5] Hans Wackernagel, Multivariate Geostatistics: An Introduction with Applications, Springer-Verlag Germany, second edition, 1998.

[6] Jean-Paul Chilès and Pierre Delfiner, Geostatistics: Modeling spatial uncertainty, John Wiley \& sons, INC., 1999. 